\title{
Long Term Solar Radiation Forecast Using Computational Intelligence Methods
}

\author{
João Paulo Coelho ${ }^{1,2}$ and José Boaventura-Cunha ${ }^{3,4}$ \\ ${ }^{1}$ ESTiG, Instituto Politécnico de Bragança, Campus de Santa Apolónia, Apartado 1134, 5301-857 Bragança, Portugal \\ ${ }^{2}$ Centre for the Research and Technology of Agro-Environmental and Biological Sciences (CITAB), Portugal \\ ${ }^{3}$ Universidade de Trás-os-Montes e Alto Douro (UTAD), Escola de Ciências e Tecnologia, Vila Real, Portugal \\ ${ }^{4}$ INESC Technology and Science (INESC TEC), Portugal \\ Correspondence should be addressed to João Paulo Coelho; jpcoelho@ipb.pt
}

Received 24 August 2014; Revised 14 November 2014; Accepted 17 November 2014; Published 11 December 2014

Academic Editor: Samuel Huang

Copyright (C) 2014 J. P. Coelho and J. Boaventura-Cunha. This is an open access article distributed under the Creative Commons Attribution License, which permits unrestricted use, distribution, and reproduction in any medium, provided the original work is properly cited.

\begin{abstract}
The point prediction quality is closely related to the model that explains the dynamic of the observed process. Sometimes the model can be obtained by simple algebraic equations but, in the majority of the physical systems, the relevant reality is too hard to model with simple ordinary differential or difference equations. This is the case of systems with nonlinear or nonstationary behaviour which require more complex models. The discrete time-series problem, obtained by sampling the solar radiation, can be framed in this type of situation. By observing the collected data it is possible to distinguish multiple regimes. Additionally, due to atmospheric disturbances such as clouds, the temporal structure between samples is complex and is best described by nonlinear models. This paper reports the solar radiation prediction by using hybrid model that combines support vector regression paradigm and Markov chains. The hybrid model performance is compared with the one obtained by using other methods like autoregressive (AR) filters, Markov AR models, and artificial neural networks. The results obtained suggests an increasing prediction performance of the hybrid model regarding both the prediction error and dynamic behaviour.
\end{abstract}

\section{Introduction}

Often the output observation of a stochastic process can not be associated with any exogenous excitation variable. These inabilities are due to several factors either because they are not known or because they can not be measured. In those circumstances, it is assumed that the process generates the observations, independently, without any outside intervention. A certain observer records the process response, usually in a regular time interval. The ultimate goal is to discover the process internal mechanism that generates the series of observations. There are an infinite number of possible mechanisms able to generate the sequence of observed values. Thus, in addition to the mechanism, or model which describes the dynamics of the process, it is necessary, in quantitative terms, to establish the quality of each of these models. The model, from all the possibilities, that exhibits the best performance, regarding the defined quality assessment function, will be the one who best describes the dynamic nature of the timeseries generating mechanism.

Autoregressive models, which only define linear relationships between past and present observations, represent one of the first attempts to explain the operating mechanism of stochastic processes [1]. However, such representations are unable to adapt to complex situations as the ones that involve nonlinear relationships between observations or even the existence of various operating regimes [2]. In this sense, the solar radiation prediction is one of these complex problems. For this reason more complex models have been gradually developed and tested. Among them are those derived from the field of computational intelligence like artificial neural networks, fuzzy logic, and support vector machines [3-5]. This paper addresses the prediction problem by using support vector regression (SVR) techniques, the autoregressive hidden Markov model, and an hybrid technique that combines both SVR and Markov chains. 


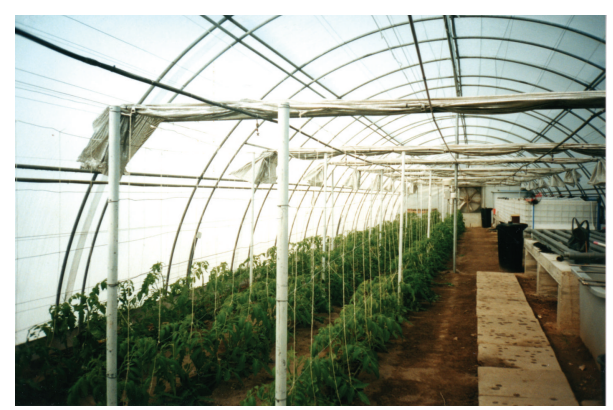

(a)

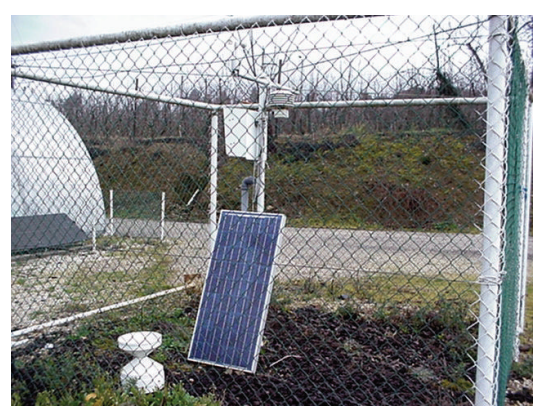

(b)

FIGURE 1: The experimental setup: a greenhouse with floor area of $210 \mathrm{~m}^{2}$ (a). Indoor temperature, relative humidity, soil temperature, and $\mathrm{CO}_{2}$ are measured. In the outside, wind speed, outside air temperature, relative humidity, and solar radiation are measured by a weather station located in the greenhouse vicinity (b).

The remaining of the paper is organized as follows: Section 2 presents an overall perspective of the solar radiation forecasting problem. Section 3 shows that the solar radiation problem can not be well described by a simple all pole filter. Increasing performance can be achieved by using an artificial neural network model. These results are presented in Section 4. In Section 5 the support vector regression paradigm is introduced and the results, concerning the solar radiation prediction for a particular day, are presented. In Section 6 the different operating process regimes are handled by hybrid strategy that combines both autoregressive models and hidden Markov models. In the sequel of this approach, Section 7 presents a new model that uses a Markov chain model and a set of support vector regression models. All the above mentioned strategies are used for solar radiation long term prediction. The results obtained, regarding two different performance indexes, for an extensive data set, are presented in Section 8. Finally, in Section 9, conclusions are drawn and some research directions are outlined.

\section{Problem Statement}

The aim of this work is to predict, as close as possible, the solar radiation dynamics during the day. This knowledge will be used, within a control loop, in order to improve the indoor temperature regulation of an agricultural building. The present problem setup includes a polyethylene cover quonset type greenhouse located at the north of Portugal. This greenhouse has a floor area of $210 \mathrm{~m}^{2}$ and is equipped with several actuators and sensors. The installed actuators are a ventilator, with a flow rate of $38000 \mathrm{~m}^{3} / \mathrm{h}$, a shadow/thermal screen, a gas heating system, with a heating power of $100416 \mathrm{KJ} / \mathrm{h}, \mathrm{CO}_{2}$ injectors, and an irrigation system. The data acquisition system stores the information provided by several sensors located inside and outside of the greenhouse. Inside the greenhouse the air temperature, relative humidity, $\mathrm{CO}_{2}$, and soil temperature, at distinct depths, are measured with a sampling period $\left(T_{s}\right)$ of one minute. Outdoor air temperature, wind speed, wind direction, and solar radiation are registered, by a weather station located in the greenhouse vicinity, at the same sampling rate. Figure 1 illustrates the experimental setup described above.

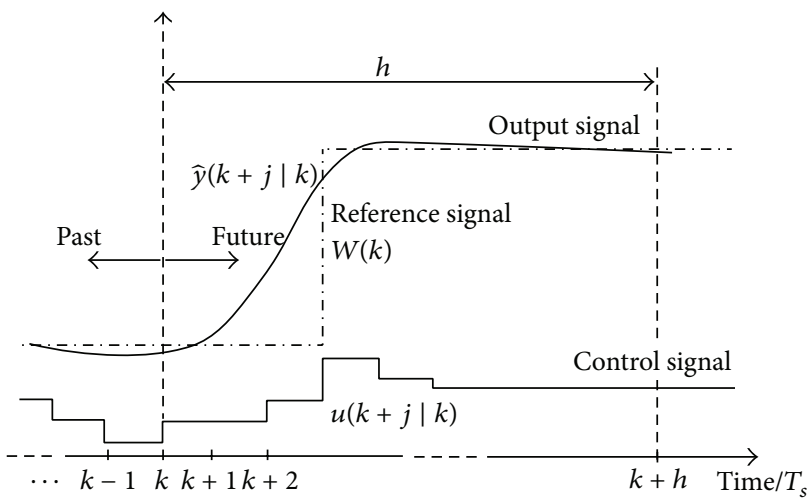

FIGURE 2: Basic strategy of a model based predictive controller. In a given time instant $k T_{s}$, the control signal sequence is forehead computed in order to minimize the future set-point tracking error (adapted from [6]).

The temperature inside the greenhouse is kept at the reference level by controlling the average power delivered to the ventilator and heating systems. The decision about which actuator is on or off and the fraction of power supplied is computed by an embedded controller. The controller software implements a model predictive control (MPC) strategy. In abstract, model predictive control comprises a collection of control methods having in common that the controller is based on the future predictions of the system behaviour, using a mathematical model of the plant [6]. MPC involves the computation of a sequence of future control values for which it is expected that the system output tracks a given input reference. The methodology underlying this type of controllers is characterized by the strategy illustrated in Figure 2. Defining a prediction horizon $h$, at a given present time instant, $k T_{s}$, the future control signal $u(k+j)$ for $j=$ $1, \ldots, h$ is computed in order to minimize the error between the simulated system response, $\widehat{y}(k+j \mid k)$, and the set-point signal $W(k+j)$. The predicted system response is computed by a plant model which has the control signals as inputs, among other possible exogenous variables. 
At each sampling instant, a set of $h$ control movements are computed. Their calculation requires solving the nonlinear optimization problem defined as [8]

$$
\begin{array}{ll}
\min _{u_{k+j}} \quad J=\sum_{j=1}^{h}[\hat{y}(k+j \mid k)-W(k+j)]^{2} \\
\text { s.t. } \quad u_{\min } \leq u(k+j) \leq u_{\max },
\end{array}
$$

where $u_{\min }$ and $u_{\max }$ refer to the actuator lower and upper bounds, generally 0 and $100 \%$ of the actuator maximum power, respectively. If $N$ different control signals are involved then there are also $N$ inequality constraints.

The control effort component can be added to the main objective function, in order to minimize the actuators wearing out. The expanded objective function has, generally, the following formulation:

$$
\begin{array}{cc}
\min _{u_{k+j}} \quad J=\sum_{j=1}^{h}[\widehat{y}(k+j \mid k)-W(k+j)]^{2} \\
\\
\quad+\gamma \sum_{j=1}^{c}[\Delta u(k+j-1)]^{2} \\
\text { s.t. } \quad u_{\min } \leq u(k+j) \leq u_{\max },
\end{array}
$$

where $\Delta u(k)=u(k)-u(k-1), c$ is the control horizon, and $\gamma$ is a tradeoff coefficient that allows choosing a more aggressive control strategy by relaxing the second right term of (2).

At each sampling instant, $h$ control movements are computed by minimizing the objective function. However, only $u(k)$ is, effectively, applied to the actuator. When a new measurement becomes available, the system parameters are updated and a new optimization problem is formulated, whose solution provides the next control movement.

This type of control strategy requires a plant model in order to obtain the predicted output value, $\widehat{y}(k)$, as a function of the computed control actions $u(k)$. If the model gives a good prediction within the control horizon then the controller performance will be high. On the other hand, if the model performance is poor, there will be a large mismatch between the predicted and the real value causing a degradation of the controller performance.

The greenhouse indoor temperature, $T_{i}$, depends on several variables such as the outdoor temperature $T_{o}$, the power supplied to the ventilation and heating systems, denoted here by $V$ and $H$, respectively, and the outdoor solar radiation $R_{o}$. Assuming that the greenhouse climate can be described by a linear system around an operating point, the greenhouse air temperature model will be described by the following firstorder autoregressive parametric equation with exogenous inputs [9].

$$
\begin{aligned}
& T_{i}(k)=\frac{\left[\beta_{1} \cdot q^{-1} \beta_{2} \cdot q^{-1} \beta_{3} \cdot q^{-1} \beta_{4} \cdot q^{-1}\right]}{1+\alpha \cdot q^{-1}} \\
& \cdot\left[\begin{array}{c}
T_{o}(k) \\
R_{o}(k) \\
V(k) \\
H(k)
\end{array}\right],
\end{aligned}
$$

where $q^{-1}$ is the backward shift operator. The model parameters $\beta_{1}$ to $\beta_{4}$ and $\alpha$ represent the partial contributions of each physical variable in the overall greenhouse air temperature.

This model is used, within a predictive control strategy, in order to infer the future value of the greenhouse indoor temperature [10]. The prediction horizon was set to sixty steps ahead, that is, a one-hour time span.

Taking into consideration the format of expression (3), in order to evaluate the indoor temperature prediction it is necessary to know the future values of both the outdoor temperature and solar radiation. The future values of $V$ and $H$ are the optimization problem solutions referred to earlier in (1) and (2). Of the two exogenous variables to be predicted, $T_{o}$ and $R_{o}$, the former has lower dynamics and is much easier to predict. On the other hand, the solar radiation has a complex behaviour, where it is possible to distinguish several operating regimes, presenting also low serial linear correlation. However, solar radiation is the external disturbance which most influences the temperature inside the greenhouse. Thus, erroneous predictions of solar radiation lead to serious forecasting indoor temperature errors which leads to bad controller performance. In this framework, the principal motivation of this work is to derive a mathematical model capable of providing adequate solar radiation predictions. The solar radiation is treated here as a time-series; that is, we assume that all observations have underline temporal structure which implies that the order of the recorded samples can not be switched.

Let $R_{o}(k)$ be the solar radiation value measured at time instant $k T_{s}$. The dependence of this value, with previous observed ones, can be established mathematically by

$$
R_{o}(k)=f\left\{R_{o}(k-1), \ldots, R_{o}(k-p)\right\}+e(k),
$$

where $f\{\cdot\}$ refers to a function that combines, in some unknown way, the past observations $R_{o}(k-1), \ldots, R_{o}(k-p)$ to generate the present observation. Additionally, $e_{k}$ refers to an unknown value generated by a process with known statistical behaviour. In this work it is assumed that the value $e_{k}$ is generated by a process normally distributed with mean 0 and variance $\sigma_{e}^{2}$.

Usually the function $f\{\cdot\}$ is unknown. It can be a simple linear combination, like autoregressive models or a linear or non-linear combination of nonlinear basis functions as in artificial neural networks or fuzzy logic models. Additionally, it can be expanded to a set of switching models just like hidden Markov models. The goal is then to find the nature of the function $f\{\cdot\}$ and, eventually, the order and structure of the lag-space. The aim is to find a function which provides the best long term prediction performance.

The maximum prediction horizon taken is sixty steps ahead and the model performance is inferred taking into consideration two indexes: the average of the root-mean squared (RMS) prediction error and the percentage of change in direction (PCD). The latter is a qualitative index representing the model ability to predict the tendency. This figure of merit is very important in the context of air temperature regulation under a model predictive controller (MPC), since the heating and ventilation requirements will be computed taking into 


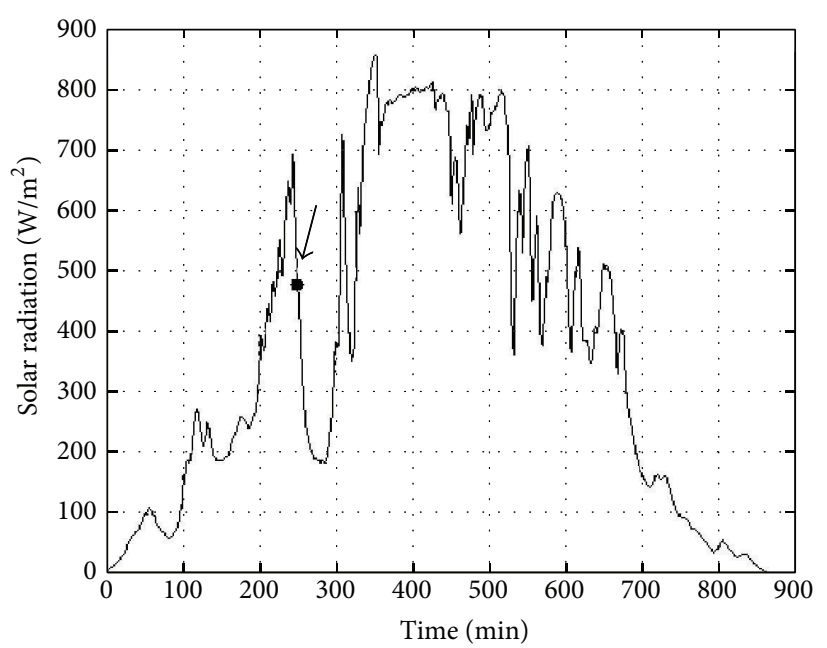

(a)

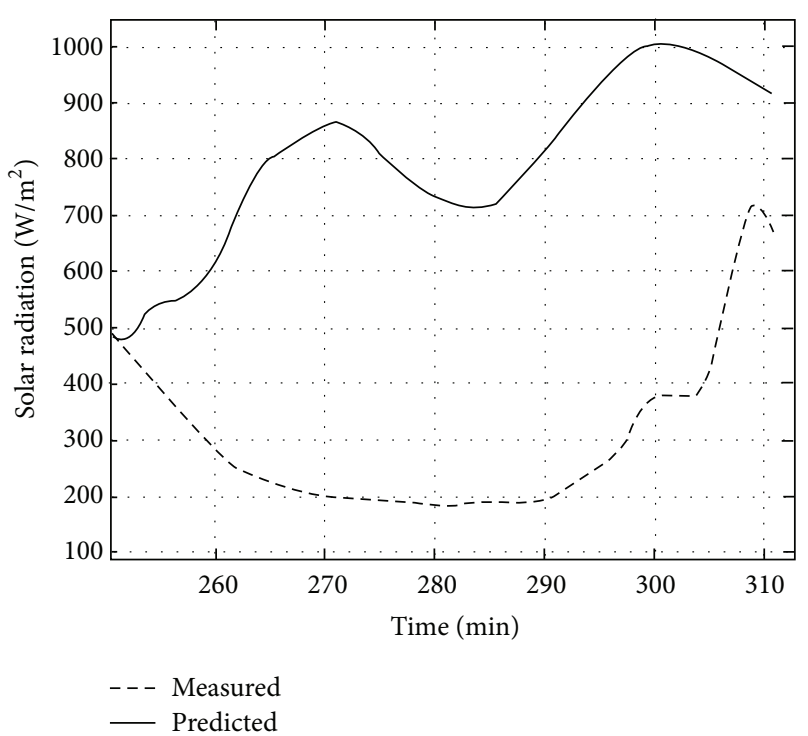

(b)

FIGURE 3: In (a), the solar radiation measured at the 8 of July of 2001. In (b), the sixty-step ahead prediction of the solar radiation, from the point identified in (a), using a 10th order AR model.

account if a heat load change is expected in the near future. The computation of both figures of merit follows:

$$
\begin{gathered}
\epsilon_{\mathrm{RMS}}=\sqrt{\frac{1}{N} \sum_{k=1}^{N}\left(R_{o}(k+h)-\widehat{R}_{o}(k+h \mid k)\right)^{2}}, \\
\epsilon_{\mathrm{PCD}}=100 \times\left(1-\left(\left(\sum_{k=2}^{N} \mid \operatorname{sign}\left(\Delta R_{o}(k+h)\right)\right.\right.\right. \\
\left.-\operatorname{sign}\left(\Delta \widehat{R}_{o}(k+h \mid k)\right) \mid\right) \\
\left.\left.\times(2(N-1))^{-1}\right)\right),
\end{gathered}
$$

where $h$ is the prediction horizon, $N$ is the total observations number, $R_{o}(k+h)$ is the solar radiation measured at time instant $(k+h) T_{s}$, and $\widehat{R}_{o}(k+h \mid k)$ is the predicted value of the solar radiation at time instant $(k+h) T_{s}$ given the information until time instant $k T_{s}$. In expression (6), the operator $\Delta$ equals $\left(1-q^{-1}\right)$ in which $q$ represents the delay operator. The signum function $\operatorname{sign}(x)$ returns 0 if $x=0$ and $x /\|x\|$ if $x \neq 0$. Hence, if all the series increments or decrements are described by the model $f\{\cdot\}$, then $\epsilon_{\mathrm{PCD}}=100 \%$. Otherwise if the model is wrong in predicting all the up and downs, then $\epsilon_{\mathrm{PCD}}=0 \%$.

In the next section the prediction results, regarding the use of an autoregressive (AR) model, are presented. For the sake of representativeness, only one day of radiation is used. Nevertheless the values obtained are coherent with those obtained using other days with the same dynamics. Section 8 presents the predicted values for longer series of data for different temporal moments along the year, four seasons.

\section{The Autoregressive Approach}

This section demonstrates the inability of AR models to provide close predictions for the solar radiation in a typical day. The parameters of a 10th order model are estimated, in a particular time instant, using the previous 80 samples after removing the linear trends. Figure 3(a) shows the prediction starting point, for the selected day. In Figure 3(b) the sixtystep ahead prediction is printed.

It can be seen that the model is unable to describe the future behaviour of the solar radiation signal. Indeed there is an early divergence between the measured and predicted signals: the model predicts an increase in radiation while, in reality, the measured signal decreases in energy. Even neglecting the discrepancy on the dynamic tendency, there is also a large difference between the prediction and measured mean values (around $500 \mathrm{~W} / \mathrm{m}^{2}$ ). To demonstrate the results persistence, another point is selected over the same radiation pattern. The new chosen point and the respective prediction result are illustrated in Figure 4. By simple visual inspection it is possible to conclude that, once again, the model was unable to follow the signal dynamic behaviour. Besides the inability to follow the signal high frequency contents, the model makes a wrong assumption about the solar radiation tendency.

Figure 5 presents the results concerning the same model but with the selected point located in the radiation pattern descendent section.

Once again the model was unable to generate acceptable results after the first (2-3) predictions. Moreover, the tendency is wrong even for the early predictions. The presented results allow us to conclude that the application of simple AR model is unsuccessful in providing good enough predictions for the solar radiation within the defined time horizon. For this reason, alternative models are tested. 


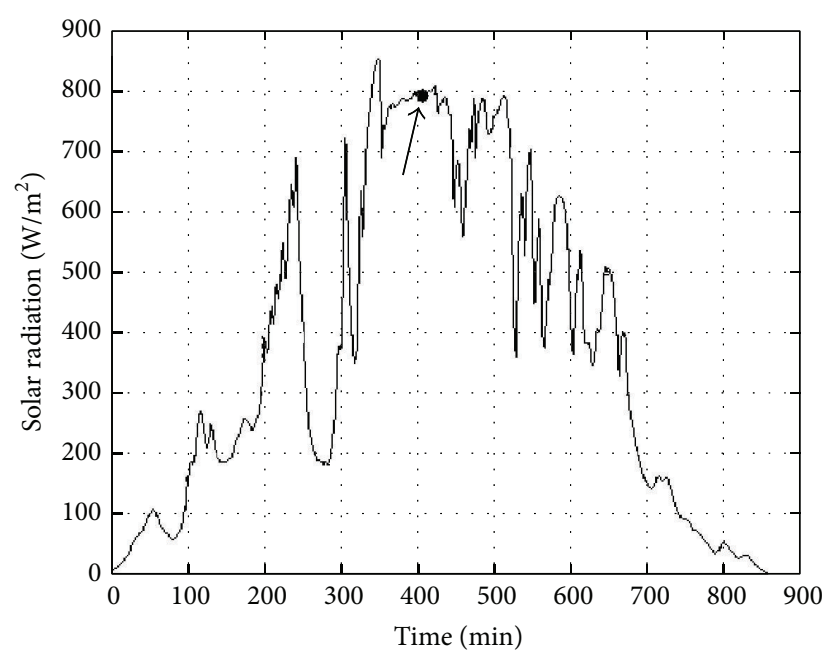

(a)

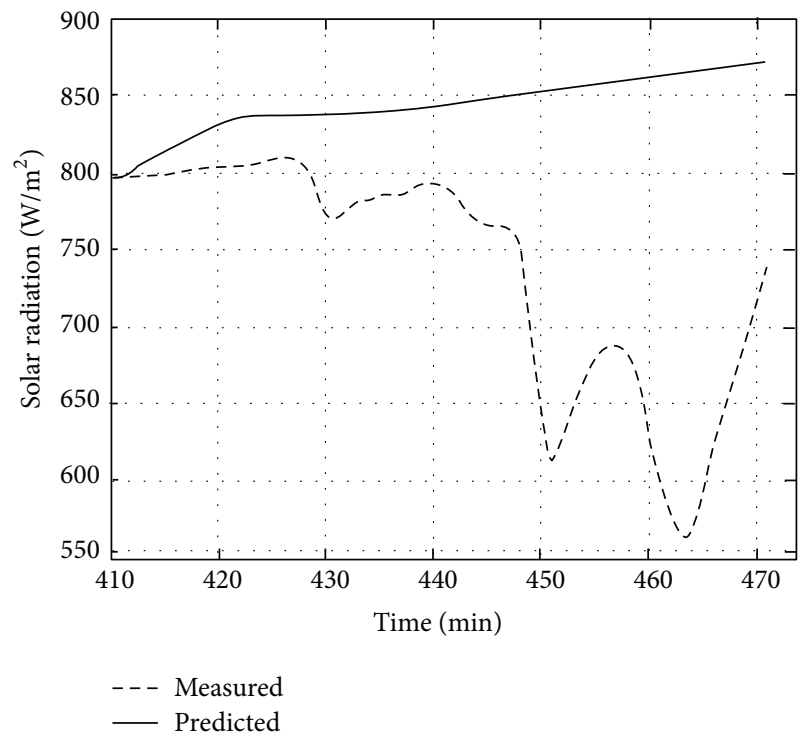

(b)

FIGURE 4: In (a), the solar radiation measured at the 8 of July of 2001. In (b), the solar radiation sixty-step ahead prediction, from the point identified in (a), using a 10th order AR model.

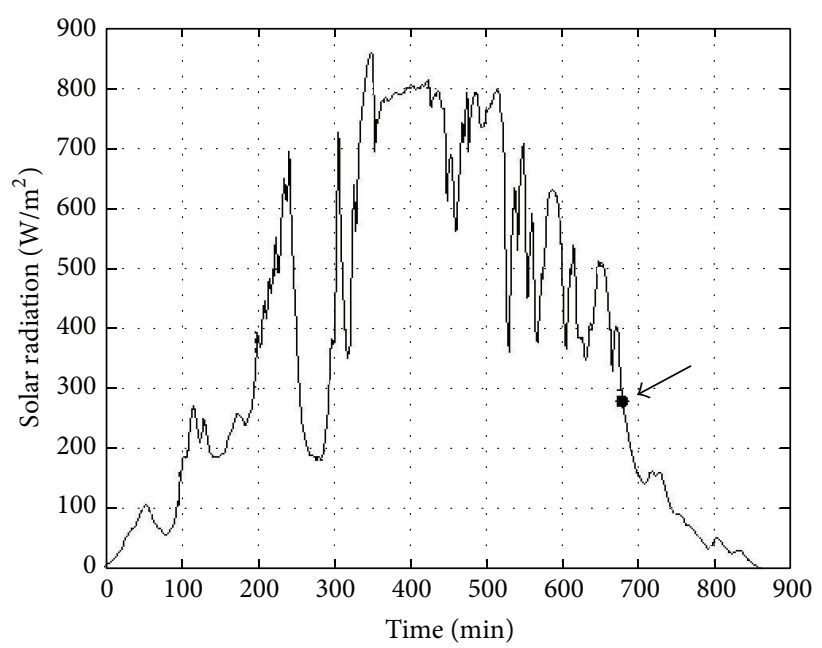

(a)

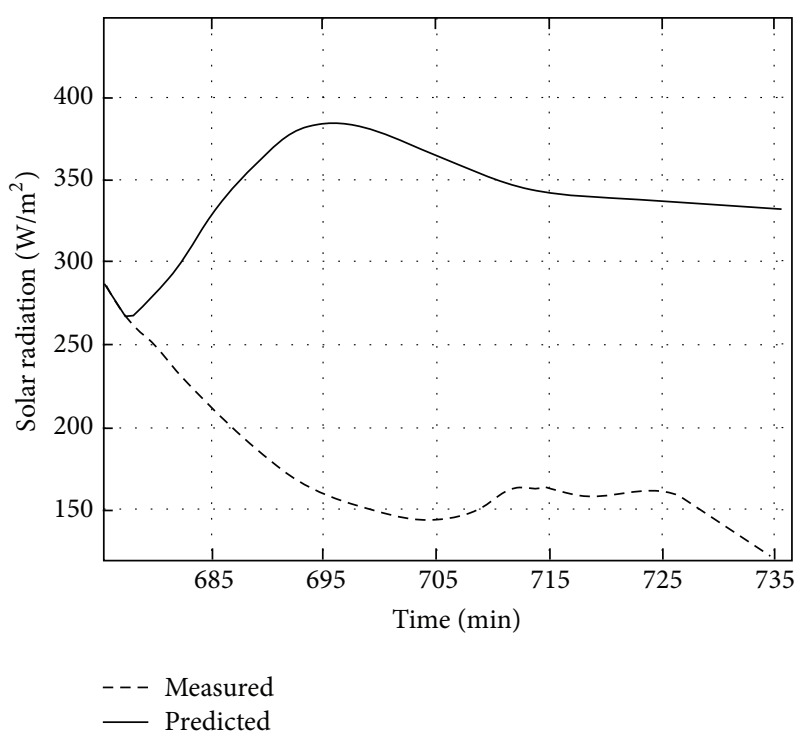

(b)

Figure 5: In (a), the solar radiation measured at the 8 of July of 2001. In (b), the solar radiation sixty-step ahead prediction, from the point identified in (a), using a 10th order AR model.

In Section 4 artificial neural network (ANN) models are used. This computational paradigm allows us to explore nonlinear relationships between observations.

\section{The Neural Network Approach}

The use of artificial neural networks for time-series prediction is not a new subject. Indeed, it is one of the most prolific modelling techniques when the nonlinear relationship between samples must be explored. In the context of solar radiation prediction, ANN models have been already used with some success [11-13]. However, even if there are improvements in the prediction quality, when compared with the AR model, the low correlation between samples and lack of signal stationarity are still two major handicaps for those models. In addition, care must be taken during the ANN training process. On one hand, the nonconvex nature of the problem tends to give only suboptimal solutions. On the other hand, an excess of model degrees-of-freedom usually results in overfitting problems. 


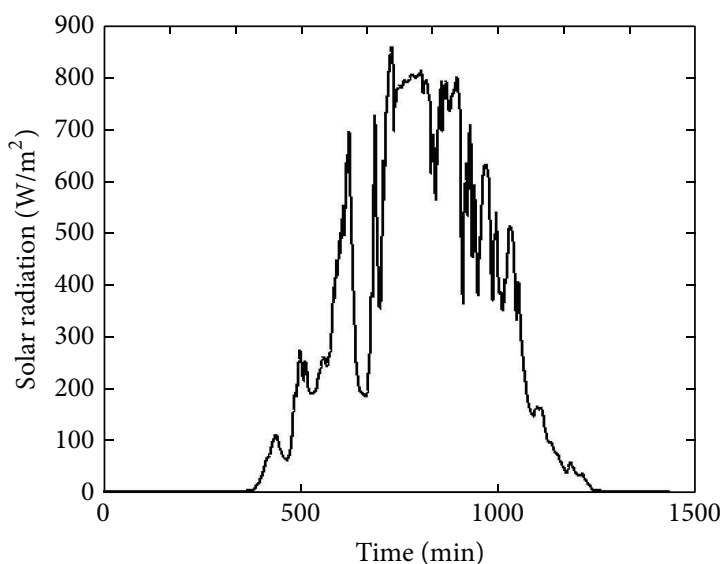

(a)
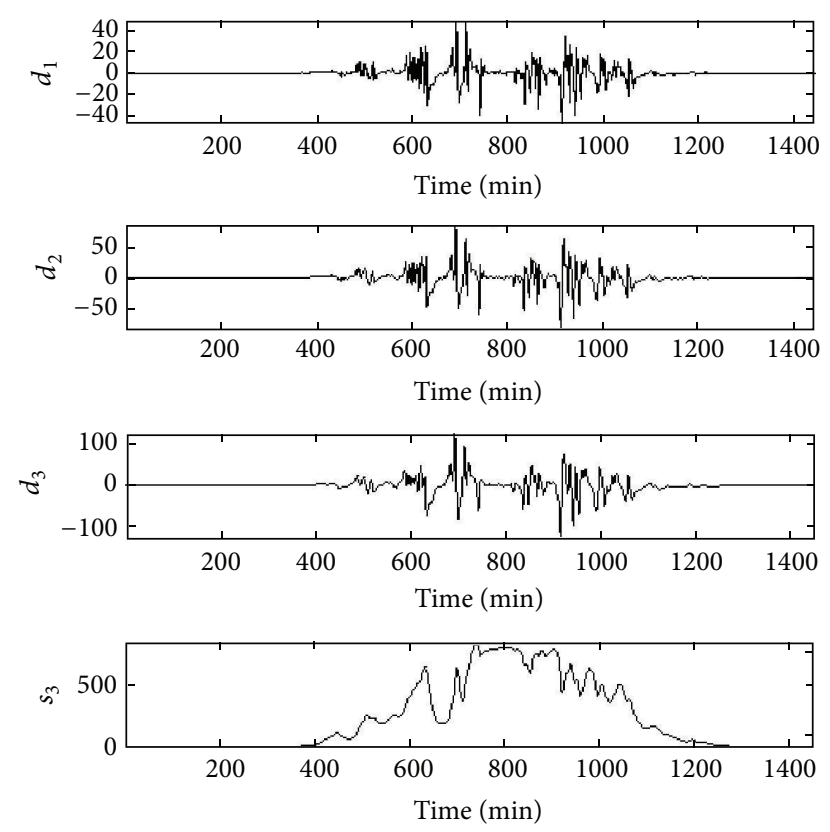

(b)

Figure 6: In (a), the solar radiation pattern used as test and, at (b), his decomposition using a filter bank. The signals $d_{1}, d_{2}$, and $d_{3}$ are obtained by high-pass filtering of the original one and $s_{3}$ from low-pass filtering.

This section presents the results concerning the use of two different ANN strategies regarding the sixty-step ahead solar radiation prediction. The first is a feedforward neural network and the latter a set of four feedforward networks each one predicting a filtered version of the data.

The ordinary feedforward neural network has a single hidden layer with five neurons each one with sigmoidal activation functions. The output layer is composed of a single neuron with linear activation function and the embedded input dimension was of 10th order. The training fase, carried out by the Levenberg-Marquardt algorithm, has used the solar radiation, collected in the past day, as estimation data.

The second nonlinear model devised was composed of four ordinary feedforward ANN. Each of them has one hidden layer with two neurons with sigmoidal activation function. The output neuron has a linear activation function and the lag-space for each network was of order five. The choice for that ANN architecture is justified in [14]. Each one of the four models is assigned to a filtered version of the signal. Those new four signals are obtained by using dilation versions of the Haar Wavelet [15].

Regarding the solar radiation of a particular day, illustrated in Figure 6(a), the decomposition of such signal, by using a Haar filter bank with three levels of detail and the firstorder tendency, is presented in Figure 6(b).

Each of the four ANN is tuned to predict one of the four decomposed signals, $d_{1}, d_{2}, d_{3}$, or $s_{3}$. In the end, the partial predictions of each network are combined in order to form the final prediction. The model with this type of structure will be labeled as ANN-WD and more detail on it can be found in [16].
TABLE 1: Results concerning the sixty-step ahead solar radiation prediction over validation data.

\begin{tabular}{lccc}
\hline & $\mathrm{AR}(10)$ & ANN & ANN-WD \\
\hline$\epsilon_{\mathrm{RMS}} / \mathrm{Wm}^{-2}$ & 126 & 123 & $\mathbf{1 1 8}$ \\
$\epsilon_{\mathrm{PCD}} / \%$ & 68 & 69 & $\mathbf{7 0}$ \\
Computational load & $<\mathbf{7 \%}$ & $28 \%$ & $100 \%$ \\
\hline
\end{tabular}

Table 1 shows the prediction results using three types of models. The first one concerns the linear AR model, presented in the previous section, and the other two described neural strategies. The presented results were computed using a time step of one minute and time prediction horizon of sixty minutes for all the day, that is, from the sunrise to the sunset. Moreover the addressed methods computational load is presented. This value is computed, under the same computational conditions, as the ratio between the elapsed time of a particular technique and the time required by the least effective method.

The obtained results suggest a slight increase in the ANN-WD model prediction capability. This improvement regards both prediction performance indexes. However, this model requires higher computational power. It is important to note that these results are obtained by the best fitted models. After several training runs with different initial solutions, the model with best prediction performance is used. The same applies to the conventional ANN. Due to the training method sensibility to the initial solution, Section 5 explores the prediction capability of another nonlinear based technique: the support vector regression model. The major 
advantage of this class of models is the training strategy used, since it involves solving a convex optimization problem.

\section{The Support Vector Regression Approach}

Support vector regression (SVR) models are a class of computational paradigms derived from support vector machines theory $[17,18]$. Like ANN, they are able to approximate, with an arbitrary precision, any well behaved linear or nonlinear function [19]. However, they have two fundamental differences when compared with neural network models. First, the training goal is not to minimize an error based criterion (like the root-mean square error) but the empirical risk. Hence the usual overfitting effect, observed in ANN, is smaller in SVR. Second, unlike ANN, the training process in SVR involves the optimization of a convex objective function subject to convex constraints [20]. For this reason the final performance of the model is independent of the initial solution [21].

In this section, the SVR with two different kernel functions is used to predict the solar radiation within the temporal range of sixty steps ahead [22]. As already stated, the solar radiation prediction, as a time-series, poses several difficulties: on the one hand the low correlation between the signal high frequency components and, on the other, the lack of stationarity.

Here two consecutive days of solar radiation data are used. The first one is for parameter estimation and the second one is for prediction. The models used are as follows.

(i) A linear AR filter with ten poles: the filter coefficients are obtained by a least squares procedure using the estimation data.

(ii) A feedforward ANN with one hidden layer with five sigmoidal neurons: the input space has dimension ten. The training procedure is carried out by using the Levenberg-Marquardt algorithm.

(iii) A neurowavelet (ANN-WD) structure is like the one described in Section 4.

(iv) Two SVR models with different kernel functions: they are a linear kernel (SVR-LK) and a Gaussian kernel (SVR-GK).

The prediction results, regarding the above enumerated models, are presented in Table 2 . The values concern only the sixty-step ahead prediction error over the validation signal. In addition, the prediction results are presented graphically in Figure 7. From this figure it can be observed that the chosen day has relevant energy in the higher part of the signal spectrum.

The graphic in the left high corner of Figure 7 shows the sixty-step ahead prediction using the AR model. Since all the poles are inside the unit circle the impulse response of this filter tends to zero with time. This characteristic is evident when the model is iterated in order to obtain predictions further in time.

The graph located at Figure 7 up right corner and lower left corner presents the results obtained by the ANN models. The first referred picture regards the ANN-WD and
TABLE 2: Sixty-step ahead prediction results, for five different models, concerning a day with some cloud disturbance.

\begin{tabular}{lccccc}
\hline & AR(10) & ANN & ANN-WD & SVR-LK & SVR-GK \\
\hline$\epsilon_{\mathrm{RMS}} / \mathrm{Wm}^{-2}$ & 41.2 & 40.9 & 39.1 & 40.5 & $\mathbf{3 8 . 5}$ \\
$\epsilon_{\mathrm{PCD}} / \%$ & 23.4 & 23.3 & $\mathbf{2 4 . 4}$ & 23.1 & 24.2 \\
\hline
\end{tabular}

the later the regular feedforward ANN. The results expressed in Table 2 regarding this two methods are consistent with the results obtained in Sections 3 and 4; that is, they provide low prediction error and better dynamic tracking characteristics when compared with the AR model. Once more the ANNWD shows an increase in performance regarding the ANN model.

The last two graphs in Figure 7 concern the support vector regression results. Taking into consideration Table 2 performance results one can conclude that the use of Gaussian kernel outperforms the linear one. Moreover the results show that the use of a SVR with linear kernel provides almost the same performance as the AR model. On the other hand, the SVR with Gaussian kernel has presented the lowest $\epsilon_{\mathrm{RMS}}$ among all the tested models.

These results allow us to conclude that, in average, the nonlinear models provide best predictions, in both performances indexes, when compared to a simple linear AR model. However their complexity is, by far, higher than that of the linear models and their performance depends on the proper choice of some training tuning parameters.

A final note on the nonlinear models used: regarding the ANN, good model weights were difficult to obtain. Several tests were performed until coefficients that would lead to models with good performance were achieved. On the other hand the values for the two tuning parameters of the SVR were obtained using the heuristics published in [7]. Due to the immunity for initial solution choices, it was simpler to obtain a good SVR model than ANN model.

So far, the techniques used consider a single model to describe the overall signal. However, there are evidences of different dynamic regimes within the data. There are obvious dynamic differences between the ascent and descent part of the day. For this reason Sections 6 and 7 explore the use of more than one model to describe the data over different regimes. The first one uses a set of AR models, whose underlining switching method is based on a Markov chain. The second one extrapolates this idea by using a set of SVR with Gaussian kernels instead of AR models.

\section{The AR Markov Model Approach}

Often it is observed that a certain model does not have the same approximation quality in all the zones of a signal produced by some unknown stochastic process. It would be possible to define alternative models that best approximate the process dynamics over particular areas. Hence it will be useful to have a set of available models, one for each dynamics, and an automatic selecting mechanisms. This mechanism will choose, among the models available, the one that best represents the process for a particular operating 

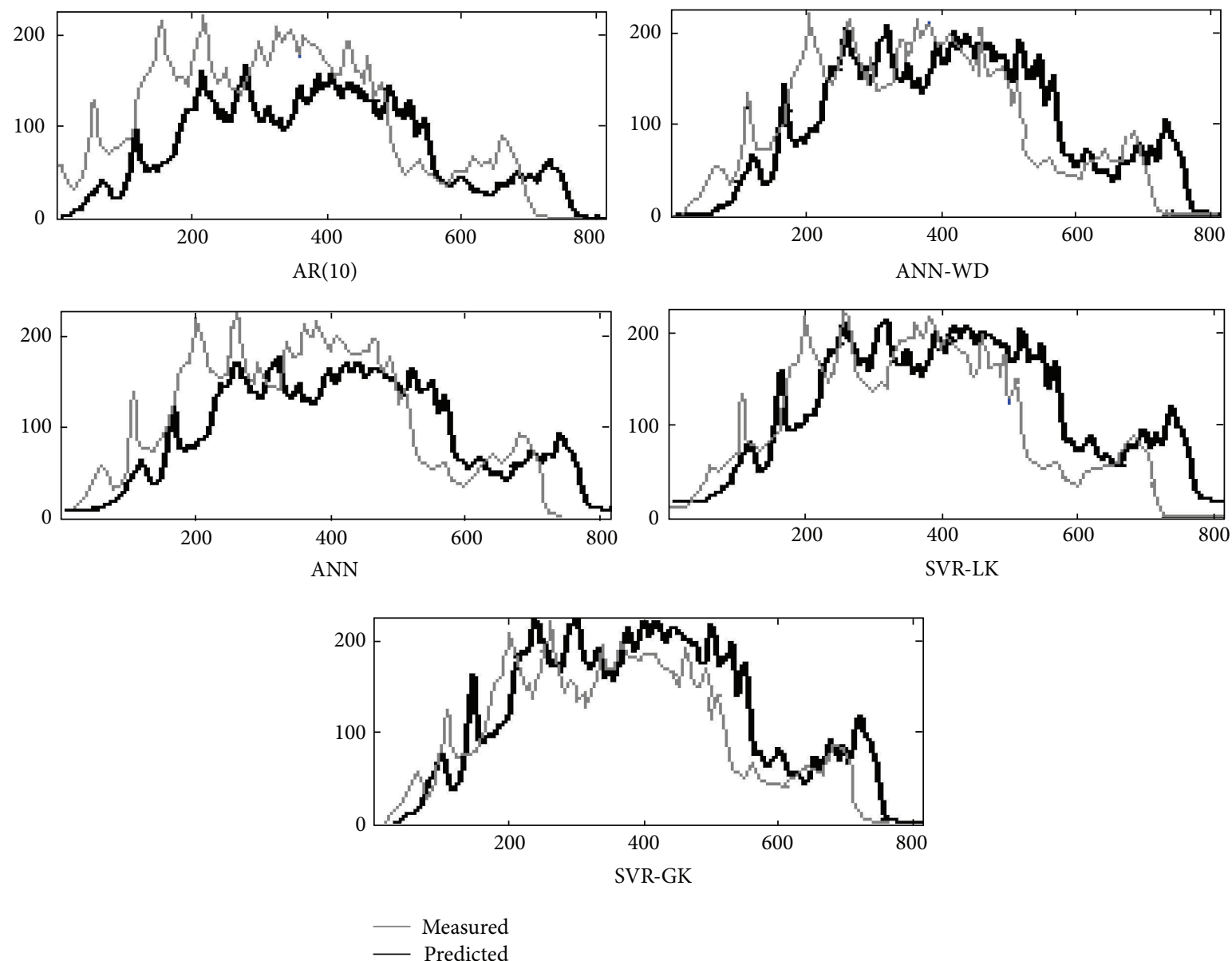

FIGURE 7: Sixty-step ahead prediction for one day of solar radiation using five different methods: a linear AR model, two distinct artificial neural networks models (ANN and ANN-WD), and two support vector regression models with different kernel functions (SVR-LK and SVR-GK). The vertical axis units are $\mathrm{W} / \mathrm{m}^{2}$ and horizontal axis is the time expressed in minutes.

point. Whenever the regime changes, a new model replaces the previous one.

For this computational paradigm, aside from the common difficulties associated with system identification problems, now there is an additional problem which is how to decide when the process regime changes. Eventually, an outer stochastic process may change the active model among the inner universe of stochastic ones. As in conventional system identification, where the model type and model order must be inferred by means of observed input/output values, the present regime knowledge, over a multiple set, must also be obtain by means of the same data. It is assumed that a stochastic process, internal to the system to model, is responsible for changing the regime. This process is not directly observable and its action can only be estimated from the only available information: present and past values of process output.

One way to emulate the regime generator mechanics is to use a Markov chain, in which the active model, at a given moment, reflects the state where the modelling process stands. Assuming a stochastic unknown process that generates the solar radiation only as a function of time then, only by direct observation of the system output data, it is possible to see that the system behaves in quite distinct forms. As an example, the different dynamics between the incident solar radiations is clear during the day and during the night. During the "night" regime, the model should switch to a constant zero while, at "day," the model should follow the fluctuations of solar radiation. Notice that, during the "day" regime, it is possible to define different subregimes. For example, the "increasing regime" that corresponds to the upward trend reflecting the radiation from the sunrise until Sun's zenith and the "decreasing regime" that corresponds to the downward behaviour from the zenith to the sunset.

In this context, this section presents the use of a multiple regime model composed by a set of autoregressive models, with different coefficient values, connected by a Markov chain which implements the model switching mechanism. A model, with this type of structure, was first presented by Hamilton and designated by Markov autoregressive model (MARM) [23, 24]. For this type of paradigm it is assumed as an $m$ state Markov chain. Each state emulates a possible system regime. Additionally, associated with each state, there is a $p$ order autoregressive model (in fact the order associated 


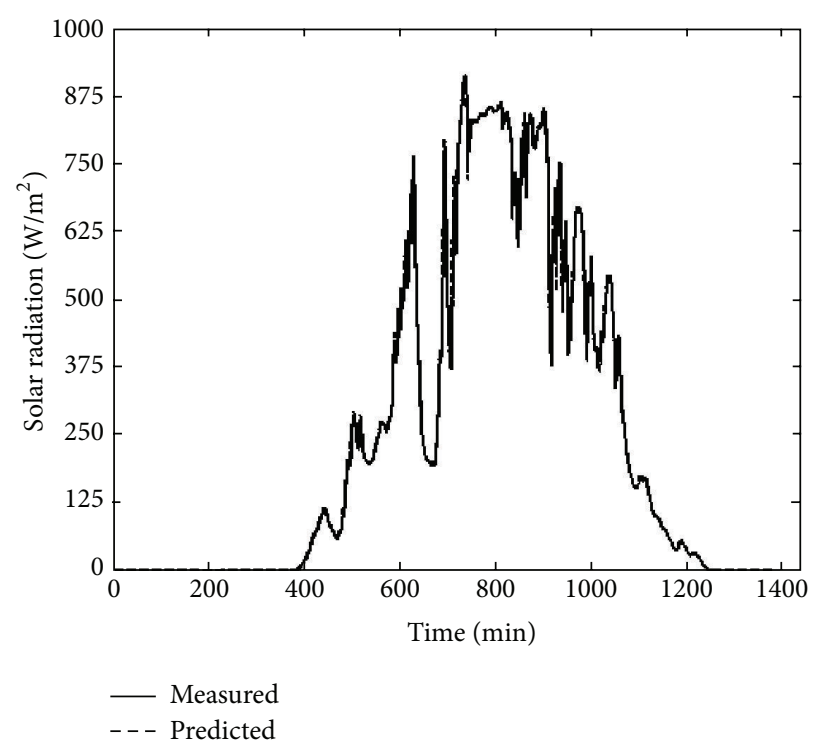

(a)

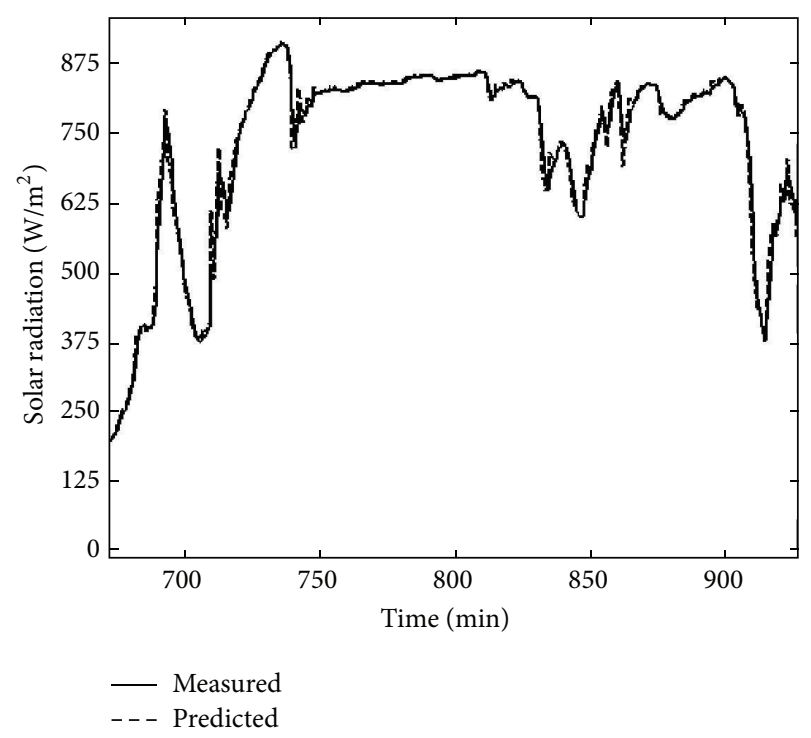

(c)

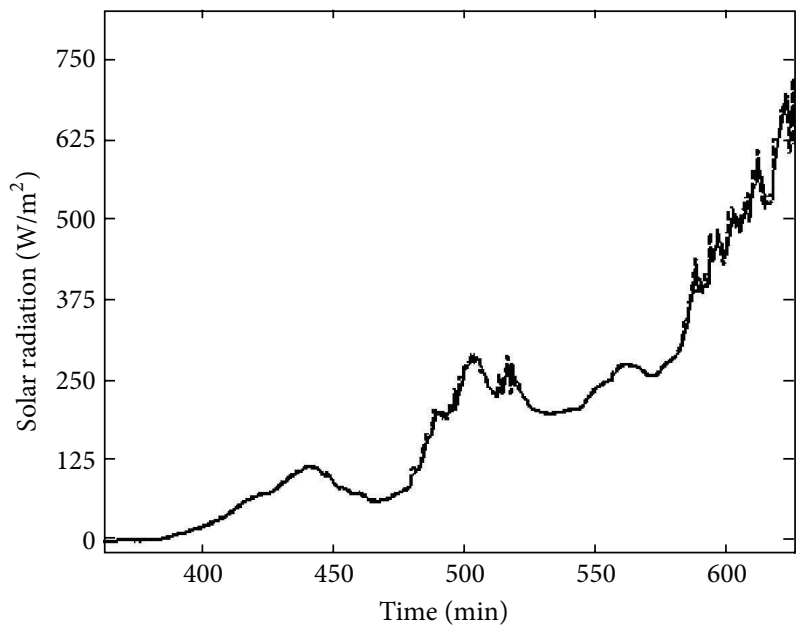

- Measured

- - - Predicted

(b)

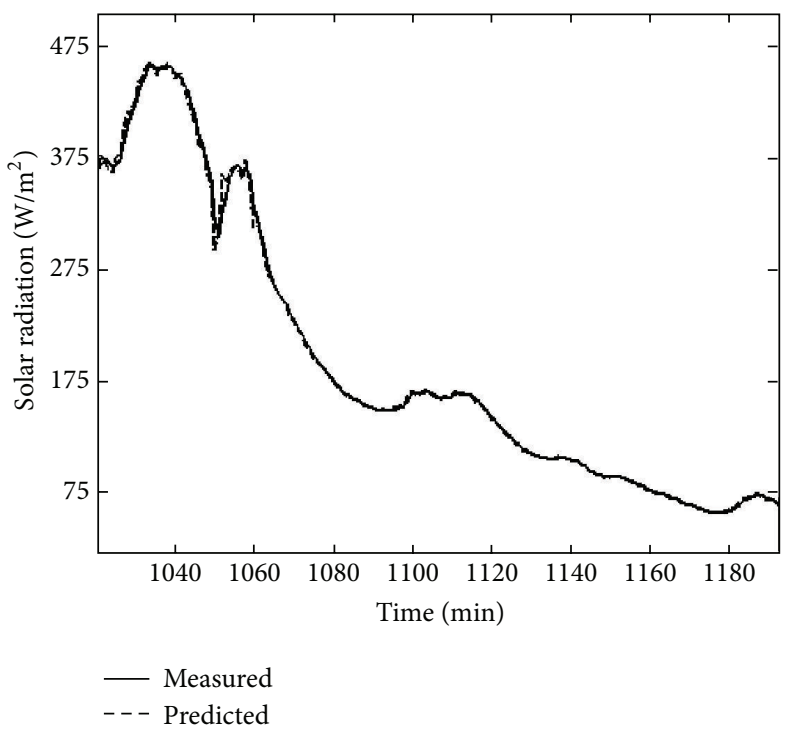

(d)

FIGURE 8: One-step ahead prediction for one day of solar radiation using a MARM with $m=3$ and $p=4$. (b), (c), and (d) represent zoomed images of (a).

with each regime can be different). If the Markov chain is at state $i$, for $1 \leq i \leq m$, then the solar radiation generating process has the following structure:

$$
R_{o}(k i)=c_{0}^{i}+c_{1}^{i} \cdot R_{o}(k-1)+\cdots+c_{p}^{i} \cdot R_{o}(k-p) .
$$

The variable $R_{o}(k \mid i)$ must be read as the observation value $R_{o}(k)$ when the process is at regime $i$. Variables $c_{0}^{i}$ to $c_{p}^{i}$ represent the $i$ th AR filter coefficients. Those values are obtained by maximizing the conditional likelihood. More details on this method can be found in [24].

A set of experiments was conducted, regarding the use of this type of model, for one- and sixty-step ahead prediction of the solar radiation. Several structures and models with distinct regimes were tested. Tables 3, 4, 5, and 6 present the prediction results obtained by using different type of MARM models. These models are distinguished, among themselves, by the number of hidden states considered and the order of the autoregressive models. Models with two, three, four, and five hidden states and for filters with two to five poles were tested. For each model, twenty experiments were executed and the results tabulated, for each situation, refer to the average of those twenty tests.

From the results presented one observes that the best model structure, in both performance indexes, has three hidden states and four poles. This statement is valid for the two prediction time horizons tested: a short term forecast of one step ahead and a long term forecast of sixty steps 


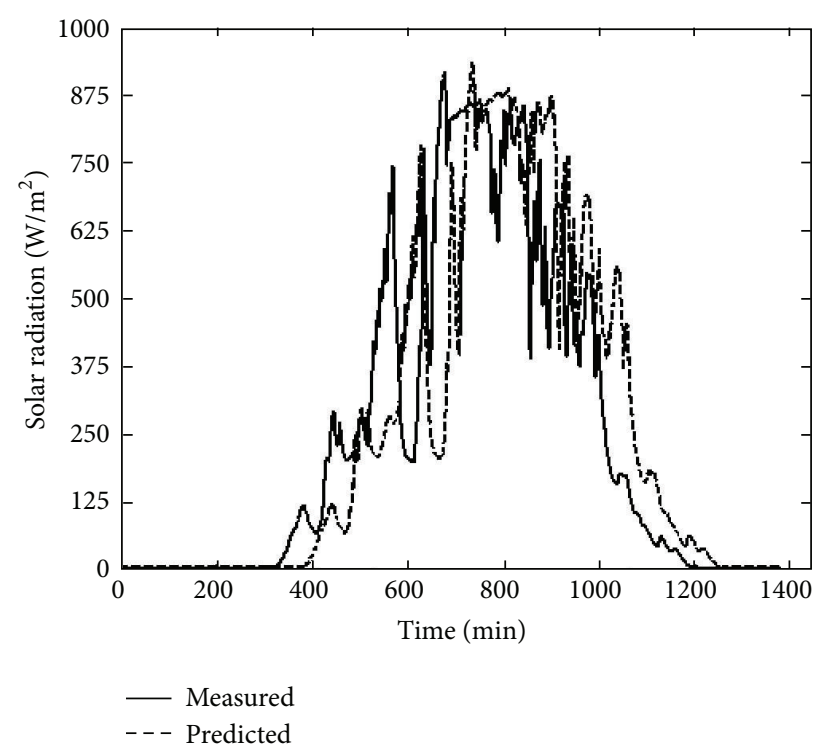

(a)

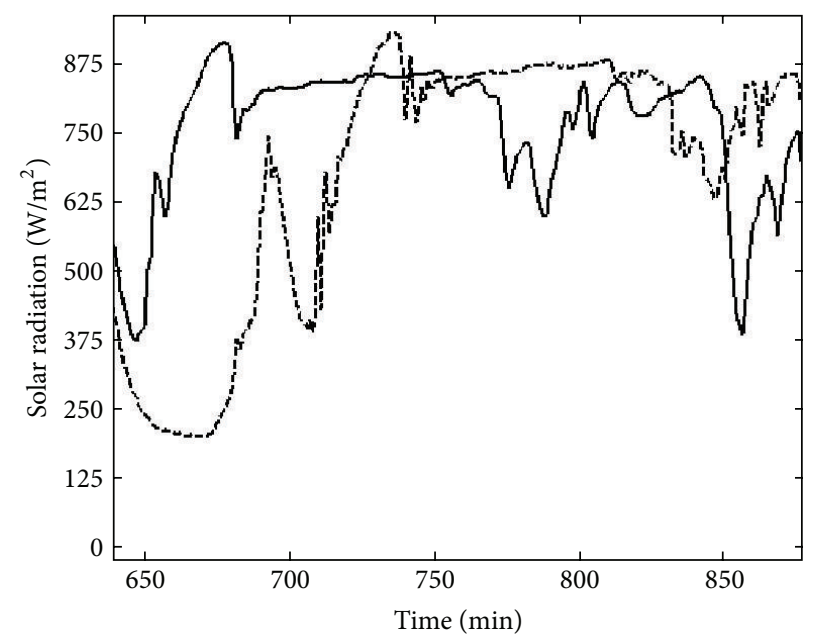

- Measured

- - - Predicted

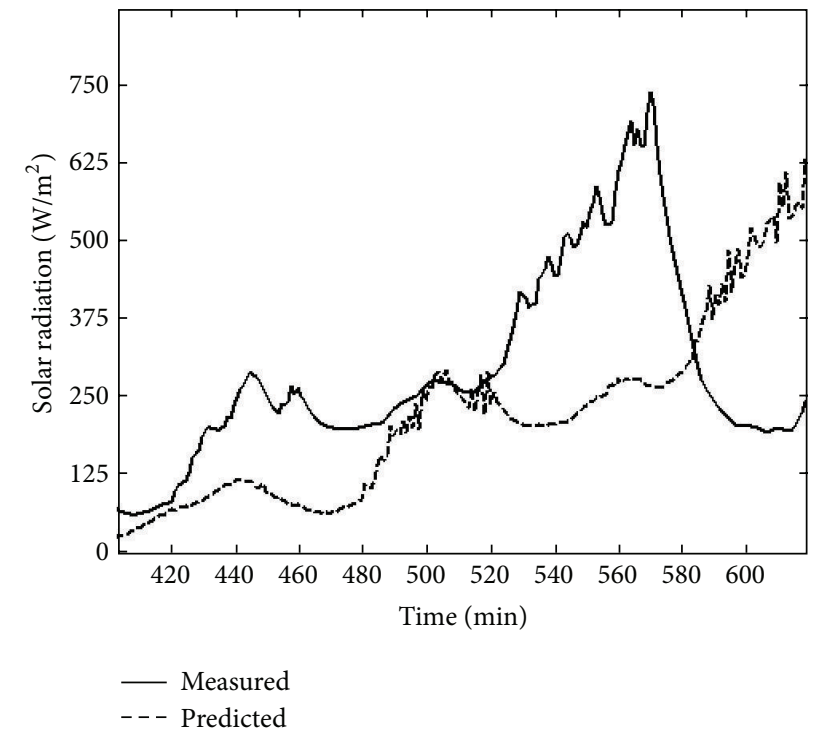

(b)

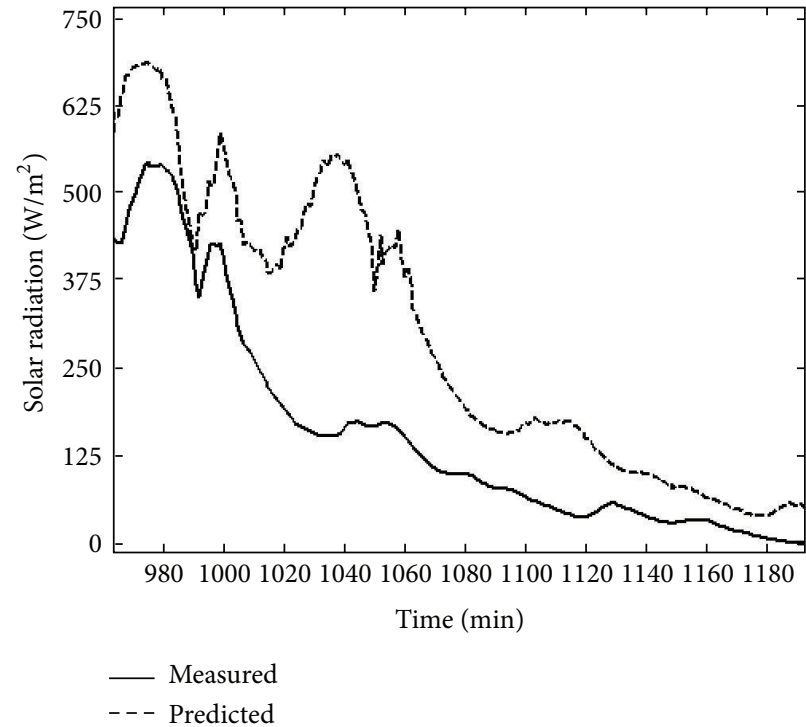

(d)

FIGURE 9: Sixty-step ahead solar radiation prediction for one day using a MARM with $m=3$ and $p=4$. (b), (c), and (d) represent zooming images of (a).

TABLE 3: Solar radiation prediction results for a two-state MARM.

\begin{tabular}{lcccccccc}
\hline Filter order $(p)$ & \multicolumn{2}{c}{2} & \multicolumn{2}{c}{3} & \multicolumn{2}{c}{4} & \multicolumn{2}{c}{5} \\
Prediction horizon/min & 1 & 60 & 1 & 60 & 1 & 60 & 1 & 60 \\
\hline$\epsilon_{\mathrm{RMS}} / \mathrm{Wm}^{-2}$ & $\mathbf{8}$ & 160 & 8.1 & $\mathbf{1 4 0}$ & 8.1 & 160 & 8.3 & 150 \\
$\epsilon_{\mathrm{PCD}} / \%$ & 82 & $\mathbf{6 5}$ & 83 & 62.8 & $\mathbf{8 5}$ & 64 & 84 & 62.4 \\
\hline
\end{tabular}

ahead. Figures 8 and 9 presented subsequently and illustrate the prediction signal profile. The former is related to the short term prediction and the latter to the long term prediction.

Comparing the results documented in Table 4 for $p=4$ and those reported in Table 1 it is possible to see that the
TABLE 4: Solar radiation prediction results for a three-state MARM.

\begin{tabular}{lcccccccc}
\hline Filter order $(p)$ & \multicolumn{2}{c}{2} & \multicolumn{2}{c}{3} & \multicolumn{2}{c}{4} & \multicolumn{2}{c}{5} \\
Prediction horizon/min & 1 & 60 & 1 & 60 & 1 & 60 & 1 & 60 \\
\hline$\epsilon_{\mathrm{RMS}} / \mathrm{Wm}^{-2}$ & 9.1 & 195 & 8.9 & 130 & $\mathbf{8 . 1}$ & $\mathbf{1 2 0}$ & $\mathbf{8 . 1}$ & 170 \\
$\epsilon_{\mathrm{PCD}} / \%$ & 84 & 60.8 & 85 & 70 & $\mathbf{8 6}$ & $\mathbf{7 1}$ & 85 & 61 \\
\hline
\end{tabular}

MARM was able to improve the $\epsilon_{\mathrm{RMS}}$, regarding the value obtained by the AR model, and the $\epsilon_{\mathrm{PCD}}$ comparatively with the ANN-WD model.

From the obtained results it seems that the direction for a good solar radiation model follows a multimodel strategy. 


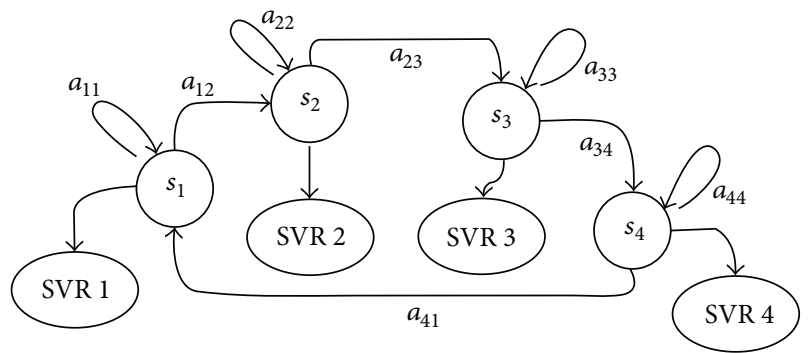

Figure 10: Topology of the hybrid SVR/Markov. Four states decide which of the present SVR to be used according to the historical data.

TABLE 5: Solar radiation prediction results for a four-state MARM.

\begin{tabular}{lcccccccc}
\hline Filter order $(p)$ & \multicolumn{2}{c}{2} & \multicolumn{2}{c}{3} & \multicolumn{2}{c}{4} & \multicolumn{2}{c}{5} \\
Prediction horizon/min & 1 & 60 & 1 & 60 & 1 & 60 & 1 & 60 \\
\hline$\epsilon_{\mathrm{RMS}} / \mathrm{Wm}^{-2}$ & 8.8 & 219.9 & $\mathbf{8 . 7}$ & 420 & 9.1 & 219.8 & 10 & $\mathbf{1 7 0}$ \\
$\epsilon_{\mathrm{PCD}} / \%$ & 81.3 & 63.1 & $\mathbf{8 5 . 1}$ & $\mathbf{6 9 . 1}$ & 85 & 65.3 & 84 & 59.5 \\
\hline
\end{tabular}

TABLE 6: Solar radiation prediction results for a five-state MARM.

\begin{tabular}{lcccccccc}
\hline Filter order $p$ & \multicolumn{2}{c}{2} & \multicolumn{2}{c}{3} & \multicolumn{2}{c}{4} & \multicolumn{2}{c}{5} \\
Prediction horizon/min & 1 & 60 & 1 & 60 & 1 & 60 & 1 & 60 \\
\hline$\epsilon_{\mathrm{RMS}} / \mathrm{Wm}^{-2}$ & 9.7 & 290 & 8.6 & 230 & $\mathbf{8 . 5}$ & $\mathbf{1 7 0}$ & 9.8 & $\mathbf{1 7 0}$ \\
$\epsilon_{\mathrm{PCD}} / \%$ & 80.5 & 57.9 & 81.2 & 59.1 & 82 & 57.9 & $\mathbf{8 2 . 3}$ & $\mathbf{5 9 . 3}$ \\
\hline
\end{tabular}

Also, as already shown, nonlinear models usually describe, in a more efficient way, the temporal sample dependence of the signal. However for the MARM strategy, even if it is a model suited for nonstationary time-series, it assumes only linear dependence over the sample space. Therefore, Section 7 presents hybrid model which interconnects a Markov chain with a set of models capable of establishing nonlinear interactions between consecutive observations.

\section{The Hybrid SVR/Markov Approach}

The previous section showed that the use of Markov autoregressive models could improve the model prediction ability. However, the MARM assumes that, within each operating regime, the dependence between observations is linear. For this reason, in this section, an alternative switching model strategy using nonlinear functions is presented. This strategy uses multiple support vector machine regression models and a Markov chain as a decision mechanism. All the SVR models considered have a Gaussian kernel function since, as demonstrated in Section 5, this type of kernel function has better predictive capability for this particular problem when compared to the linear one.

The process of defining the support vectors involves the partition of the training data into four segments. Each segment represents a particular part of the day: the absence of radiation, during the ascending part of the day, the descending part of the day and around the peak of radiation, where the first derivative has a lower value. Subsequently four SVR models are fitted, one for each regime.
TABLE 7: Results concerning the sixty-step ahead solar radiation prediction over validation data.

\begin{tabular}{lccccc}
\hline & AR(10) & ANN & ANN-WD & MARM & SVR/Markov \\
\hline$\epsilon_{\mathrm{RMS}} / \mathrm{Wm}^{-2}$ & 126 & 123 & 118 & 120 & $\mathbf{1 1 2}$ \\
$\epsilon_{\mathrm{PCD}} / \%$ & 68 & 69 & 70 & 71 & $\mathbf{7 9 . 1}$ \\
\hline
\end{tabular}

The active SVR, in a particular time instant, is defined by a four-state Markov chain with a Bakis topology [25]. Hence, there is a single propagation direction between adjacent states. Figure 10 illustrates this situation. Each round object refers to one different state designated by $s_{1}$ to $s_{4}$. The ellipse type shapes concern the four different SVR models and the arrows, linking the states, represent the transition with arbitrary probability $a_{i j}$ for $i=1, \ldots, 4$ and $j=1, \ldots, 4$. Note that, due to stochastic constraints, $0 \leq a_{i j} \leq 1$ and $a_{i j}+a_{i j}=1$. These probabilities are estimated, using a set of the previous ten days, by the Baum-Welch algorithm [26, 27].

The prediction is made under two different hierarchical levels. The first involves the prediction of the state sequence and the second the solar radiation prediction made by the model pointed out by the state sequence. This type of strategy was applied to predict the solar radiation for a particular day already presented in above sections. The performance results concerning the prediction horizon of sixty steps ahead are presented in Table 7. For convenience the results obtained by the previous methods are repeated in the same table.

Figure 11 presents the prediction results with the hybrid SVR/Markov model. This figure is partitioned in four graphs. Figure 11(a) represents the overall prediction for the selected day and the other three zooming zones regarding the referred graphic. The predicted signal sections selected represent the prediction at the beginning of the day, at the middle of the day, and around the end of the day.

The obtained results, even if just for a particular day, show clearly that the hybrid SVR/Markov model was able to give a best performance, in both defined indexes, when compared to the other tested methods. Section 8 shows that this predominance, over the other methods, is coherent and insensible to the selected day.

\section{Solar Radiation Prediction Results for Several Days}

In order to be able to present a graphical output concerning the obtained results, the earlier sections lie in the application of several model techniques for only a particular day. Even if the spectral content of the chosen day represents those of a regular day, in this section the above mentioned computational models were used for solar radiation over a larger data set. Whenever required, the model parameters estimations were obtained, using the training methodology discussed in the preceding sections, over the previous day.

The obtained results are presented in Tables 8, 9, 10, and 11 . Each table concerns the results obtained, for the two criteria, by averaging the prediction results over ten days for all the year, four seasons. 


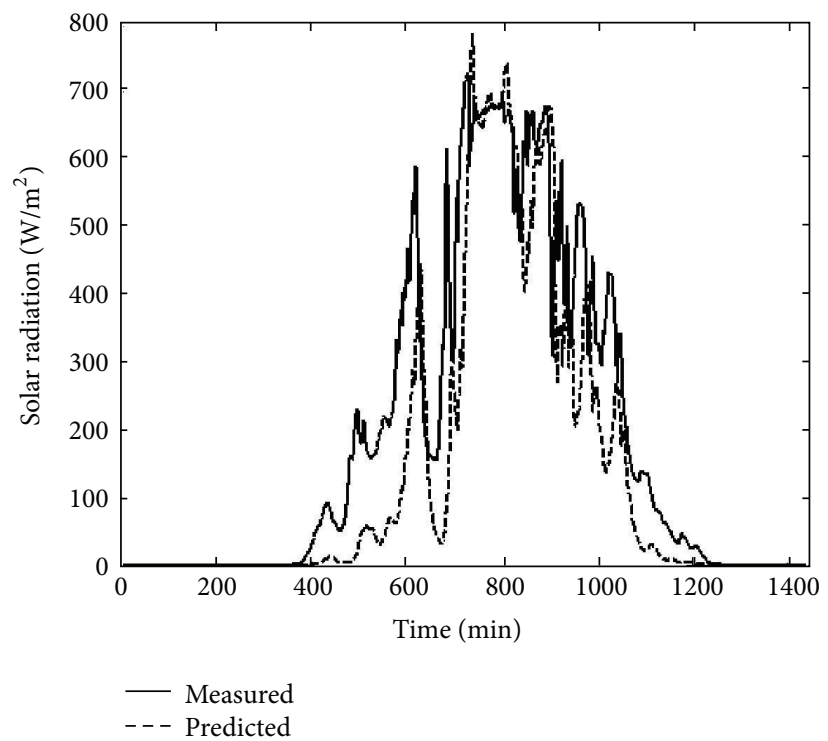

(a)

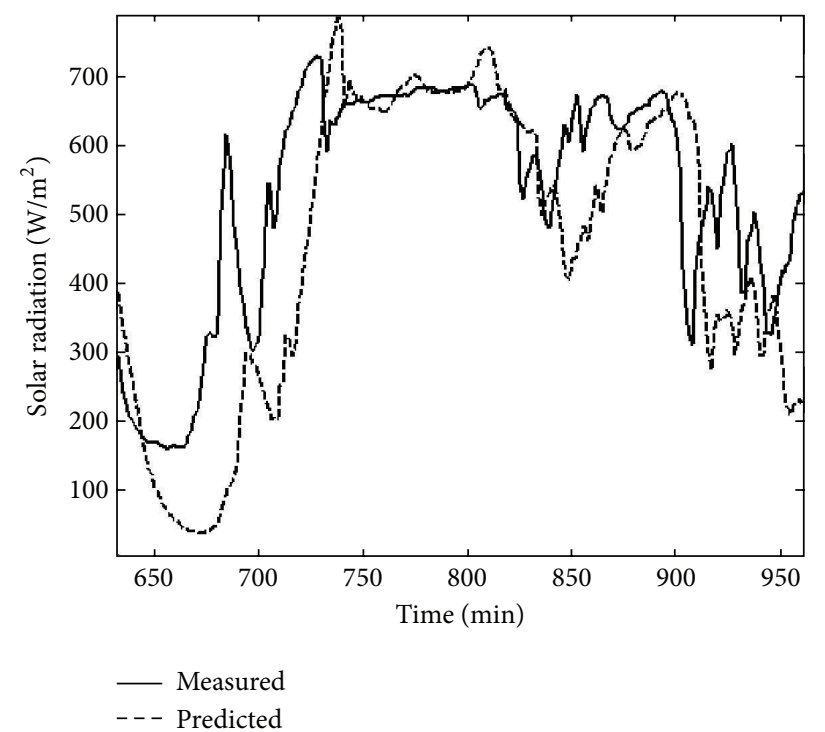

(c)

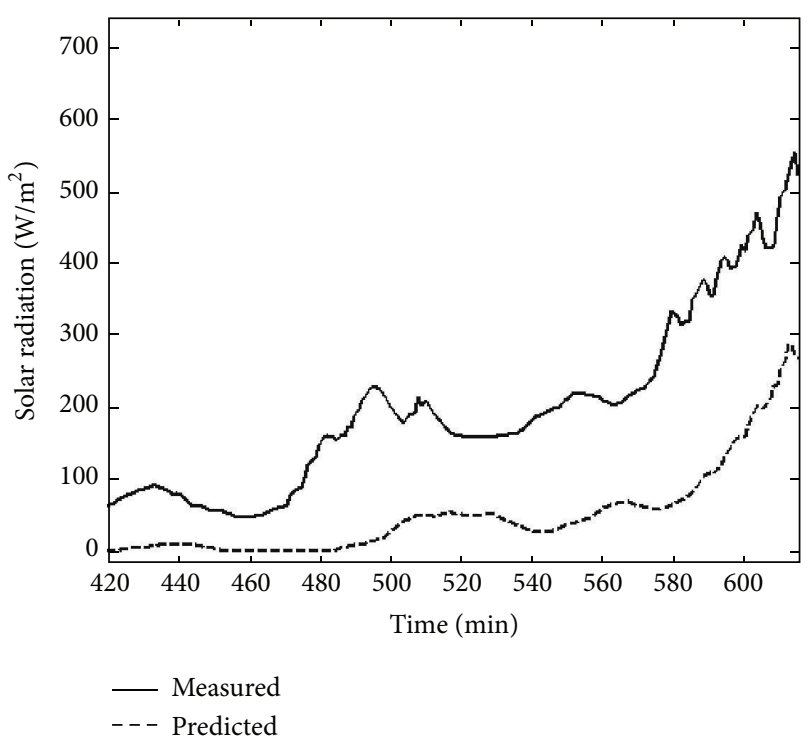

(b)

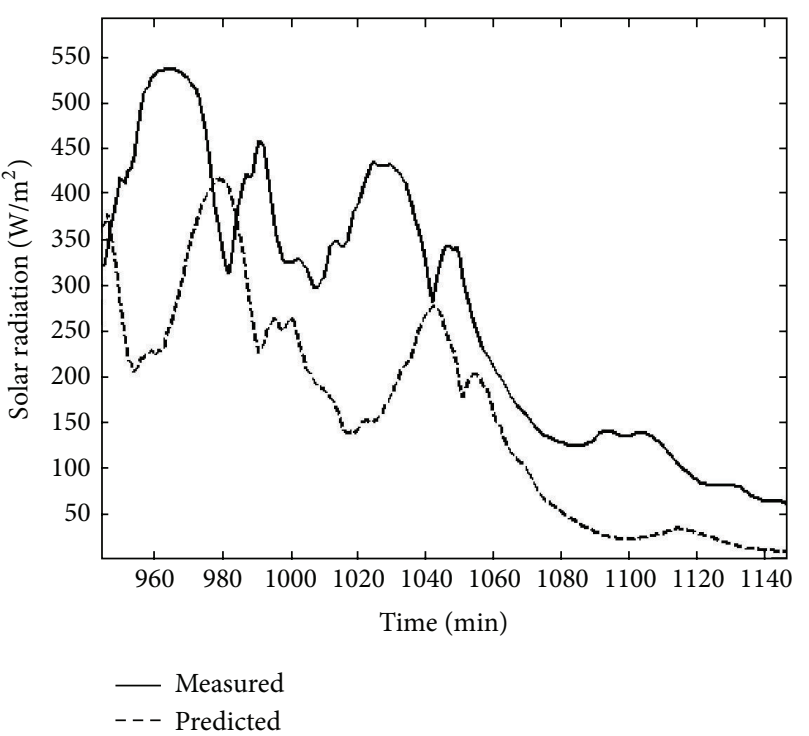

(d)

FIGURE 11: Sixty-step ahead solar radiation prediction for one day using a hybrid SVR/Markov with four states. (b), (c), and (d) represent zooming images of (a).

TABLE 8: Obtained average prediction values for ten days selected from April to May of 2000 (spring).

\begin{tabular}{lcccccccccccc}
\hline Model & \multicolumn{2}{c}{ AR(10) } & \multicolumn{2}{c}{ ANN } & \multicolumn{2}{c}{ ANN-WD } & \multicolumn{2}{c}{ SVR-GK } & \multicolumn{2}{c}{ MARM } & \multicolumn{2}{c}{ SVR/Markov } \\
Horizon/min & 1 & 60 & 1 & 60 & 1 & 60 & 1 & 60 & 1 & 60 & 1 & 60 \\
\hline$\epsilon_{\mathrm{RMS}} / \mathrm{Wm}^{-2}$ & 8.35 & 113.1 & 8.2 & 112.6 & 8.2 & 108.1 & 8.1 & 107.6 & 8.31 & 109.9 & $\mathbf{7 . 9 8}$ & $\mathbf{1 0 4 . 8}$ \\
$\epsilon_{\mathrm{PCD}} / \%$ & 87.7 & 73.3 & 88.1 & 74.7 & 88.4 & 75.7 & 88.7 & 76.1 & 88.1 & 75.2 & $\mathbf{9 0 . 3}$ & $\mathbf{7 9 . 3}$ \\
\hline
\end{tabular}

TABLE 9: Obtained average prediction values for ten days selected from July to August of 2000 (summer).

\begin{tabular}{lcccccccccccc}
\hline Model & \multicolumn{2}{c}{ AR(10) } & \multicolumn{2}{c}{ ANN } & \multicolumn{2}{c}{ ANN-WD } & \multicolumn{2}{c}{ SVR-GK } & \multicolumn{2}{c}{ MARM } & \multicolumn{2}{c}{ SVR/Markov } \\
Horizon/min & 1 & 60 & 1 & 60 & 1 & 60 & 1 & 60 & 1 & 60 & 1 & 60 \\
\hline$\epsilon_{\mathrm{RMS}} / \mathrm{Wm}^{-2}$ & 8.43 & 103.8 & 8.3 & 103.5 & 8.3 & 100.6 & 8.2 & 100.2 & 8.39 & 101.1 & $\mathbf{8 . 1 6}$ & $\mathbf{9 3 . 7}$ \\
$\epsilon_{\mathrm{PCD}} / \%$ & 87.5 & 72.1 & 87 & 72.6 & 88 & 74.5 & 87.8 & 75.1 & 87.6 & 73.8 & $\mathbf{8 8 . 7}$ & $\mathbf{7 5 . 5}$ \\
\hline
\end{tabular}


TABLE 10: Obtained average prediction values for ten days selected from October to November of 2000 (autumn).

\begin{tabular}{lcccccccccccc}
\hline Model & \multicolumn{2}{c}{ AR(10) } & \multicolumn{2}{c}{ ANN } & \multicolumn{2}{c}{ ANN-WD } & \multicolumn{2}{c}{ SVR-GK } & \multicolumn{2}{c}{ MARM } & \multicolumn{2}{c}{ SVR/Markov } \\
Horizon $/ \mathrm{min}$ & 1 & 60 & 1 & 60 & 1 & 60 & 1 & 60 & 1 & 60 & 1 & 60 \\
\hline$\epsilon_{\mathrm{RMS}} / \mathrm{Wm}^{-2}$ & 2.22 & 32.4 & 2.18 & 31.6 & 2.12 & 30.83 & 2.1 & 30.2 & 2.19 & 31.1 & $\mathbf{1 . 9 8}$ & $\mathbf{2 9 . 9}$ \\
$\epsilon_{\mathrm{PCD}} / \%$ & 91 & 82.4 & 91.4 & 84.1 & 91.8 & 87.2 & 92.6 & 86.5 & 91.9 & 83.3 & $\mathbf{9 2 . 8}$ & $\mathbf{8 7 . 8}$ \\
\hline
\end{tabular}

TABLE 11: Obtained average prediction values for ten days selected from January to February of 2000 (winter).

\begin{tabular}{lcccccccccccc}
\hline Model & \multicolumn{2}{c}{ AR(10) } & \multicolumn{2}{c}{ ANN } & \multicolumn{2}{c}{ ANN-WD } & \multicolumn{2}{c}{ SVR-GK } & \multicolumn{2}{c}{ MARM } & \multicolumn{2}{c}{ SVR/Markov } \\
Horizon $/ \mathrm{min}$ & 1 & 60 & 1 & 60 & 1 & 60 & 1 & 60 & 1 & 60 & 1 & 60 \\
\hline$\epsilon_{\mathrm{RMS}} / \mathrm{Wm}^{-2}$ & 2.69 & 53.8 & 2.68 & 54.1 & 2.6 & 52.4 & 2.5 & 52.3 & 2.51 & 52.7 & $\mathbf{2 . 4 6}$ & $\mathbf{5 2 . 2}$ \\
$\epsilon_{\mathrm{PCD}} / \%$ & 87 & 77 & 86.5 & 76.8 & 87.4 & 78.8 & 88.1 & 78.1 & 88 & $\mathbf{7 8 . 8}$ & $\mathbf{8 8 . 2}$ & $\mathbf{7 9 . 1}$ \\
\hline
\end{tabular}

In the first row of Tables 8 and 9 the prediction error, regarding two different time horizons, is presented. Regarding the one step ahead, the results obtained, by the tested models, are all very similar to the one obtained by the autoregressive model. However the differences between models become more apparent when the forecasting horizon is increased. For the case of sixty steps ahead, the prediction error has a lower value for the hybrid SVR/Markov model when comparing to the remaining values. However the value achieved by this method is followed very closely by other models, in particular the SVR with simple Gaussian kernel (SVR-GK) and artificial neural network with wavelet decomposition (ANN-WD).

Regarding the dynamic behaviour results, presented in the second row of Tables 8 and 9, the values obtained by the SVR/Markov are higher than the values obtained by its competitors. This increase in performance is due to the four different specialized SVR models. It is worthwhile to remark that the above conclusions are valid for the four considered seasonal samples.

In Section 9 additional comments, regarding the obtained results, are presented. In addition, it is pointed out as model improvements and trends for further research.

\section{Conclusions and Further Work}

In this work the solar radiation long term prediction, represented as a time-series, was carried out by a set of linear and nonlinear models. The close knowledge of future values of this variable will be fundamental to improve the greenhouse indoor temperature controller performance.

The solar radiation prediction, within this framework, is a very complex problem in part not only due to the low correlation between samples, especially in days with high radiation variability, but also due to the nonstationary behaviour of the generating process. Moreover, due to the large prediction horizon addressed, small model changes can result in large prediction errors. This is due to the feedback nature of the prediction process.

For this reason, a set of models was tested: linear autoregressive, nonlinear autoregressive, and support vector models. In addition an alternative computational paradigm, evolving SVR and Markov chains, was proposed. All the models were applied to large data sets concerning the solar radiation collected in several days distributed along the year.

The results presented indicated that the best tested model was the one that combines support vector regression and Markov chains. The relevance of this model becomes conspicuous for increasing prediction horizons. The advantage of this model is its capability to handle processes with several operating regimes and nonlinear behaviour. Even if the SVR/Markov results are better than those obtained by other strategies, it is believed that an additional increase in performance can be achieved by using more sophisticated model training methods. For now the SVR and the Markov chain training are handled separately. Therefore it is proposed, as a trend for future research, to change the training method of this model. An alternative way would be to transfer the technique that Hamilton has used for training MARM and adapt it to this new model. That is, both the parameters chain and support vector can be evolved simultaneously. In this way one could eliminate the need for segmentation and expert assessment of the data. In addition, in the current problem, the number of system states has been defined empirically. So another research direction is the development of a technique that enables the automatic selection of the best number of hidden regimes without a full-factorial experiment.

\section{Conflict of Interests}

The authors declare that there is no conflict of interests regarding the publication of this paper.

\section{References}

[1] G. Box and G. Jenkins, Time Series Analysis: Forecasting and Control, 1970.

[2] A. L. S. Maia, F. D. A. T. de Carvalho, and T. B. Ludermir, "Forecasting models for interval-valued time series," Neurocomputing, vol. 71, no. 16-18, pp. 3344-3352, 2008.

[3] P. G. Zhang, "Time series forecasting using a hybrid ARIMA and neural network model," Neurocomputing, vol. 50, pp. 159$175,2003$.

[4] W. Ji and K. C. Chee, "Prediction of hourly solar radiation using a novel hybrid model of ARMA and TDNN," Solar Energy, vol. 85, no. 5, pp. 808-817, 2011. 
[5] S. X. Chen, H. B. Gooi, and M. Q. Wang, "Solar radiation forecast based on fuzzy logic and neural networks," Renewable Energy, vol. 60, pp. 195-201, 2013.

[6] E. Camacho and C. Bordons, Model Predictive Control in the Process Industry, Springer, 1994.

[7] V. Cherkassky and Y. Ma, "Practical selection of SVM parameters and noise estimation for SVM regression," Neural Networks, vol. 17, no. 1, pp. 113-126, 2002.

[8] D. W. Clarke, C. Mohtadi, and P. S. Tuffs, "Generalized predictive control-Part I. the basic algorithm," Automatica, vol. 23, no. 2, pp. 137-148, 1987.

[9] J. Boaventura Cunha, C. Couto, and A. E. B. Ruano, "A greenhouse climate multivariable predictive controller," Acta Horticulturae, vol. 534, pp. 269-276, 2000.

[10] B. Nielsen and H. Madsen, "Predictive control of air temperature in greenhouses," in Proceedings of the IFAC 13th Triennial World Congress, pp. 399-404, 1996.

[11] J. P. Coelho, Comparative study of techniques for modeling and forecasting time series [M.S. thesis], Universidade de Trás-osMontes e Alto Douro, 2003.

[12] P. Ferreira and A. Ruano, "Predicting solar radiation with rbf neural networks," in Proceedings of the 7th Portuguese Conference on Automatic Control (CONTROLO '04), 2004.

[13] E. M. Crispim, P. M. Ferreira, and A. E. Ruano, "Solar radiation prediction using RBF Neural Networks and cloudiness indices," in Proceedings of the International Joint Conference on Neural Networks (IJCNN '06), pp. 2611-2618, July 2006.

[14] J. P. Coelho, Solar radiation prediction by softcomputing techniques [Ph.D. thesis], Universidade de Trás-os-Montes e Alto Douro, 2011.

[15] J. Walker, A Primer on Wavelets and their Scientific Applications, Chapman \& Hall/CRC, 1999.

[16] J. P. Coelho, J. Boaventura Cunha, and P. B. de Moura Oliveira, "Solar radiation prediction using wavelet decomposition," in Proceedings of the CONTROLO 8th Portuguese Conference on Automatic Control, 2008.

[17] V. Vapnik, S. Golowich, and A. Smola, "Support vector method for function approximation, regression estimation and signal processing," in Neural Information Processing Systems Foundation, 1996.

[18] V. Vapnik, Statistical Learning Theory, John Wiley \& Sons, New York, NY, USA, 1998.

[19] B. Hammer and K. Gersmann, "A note on the universal approximation capability of support vector machines," Neural Processing Letters, vol. 17, no. 1, pp. 43-53, 2003.

[20] N. Cristianini and J. Shawe-Taylor, Support Vector Machines and other Kernelbased Learning Methods, Cambridge University Press, 2000.

[21] S. Boyd and L. Vandenberghe, Convex Optimization, Cambridge University Press, Cambridge, UK, 2004.

[22] J. P. Coelho, J. Boaventura Cunha, and P. B. D. Oliveira, "Greenhouse heat load prediction using a support vector regression model," in Soft Computing Models in Industrial and Environmental Applications, 5th International Workshop (SOCO 2010), vol. 73 of Advances in Intelligent and Soft Computing, pp. 111-117, 2010.

[23] J. D. Hamilton, "Analysis of time series subject to changes in regime," Journal of Econometrics, vol. 45, no. 1-2, pp. 39-70, 1990.

[24] J. D. Hamilton, Time Series Analysis, Princeton University Press, Princeton, NJ, USA, 1994.
[25] L. R. Rabiner and B.-H. Juang, "An introduction to hidden markov models," IEEE ASSP magazine, vol. 3, no. 1, pp. 4-16, 1986.

[26] L. Welch, "Hidden markov models and the baum-welch algorithm," IEEE Information Theory Society Newsletter, vol. 53, no. 1, pp. 10-13, 2003.

[27] L. E. Baum and T. Petrie, "Statistical inference for probabilistic functions of finite state Markov chains," Annals of Mathematical Statistics, vol. 37, pp. 1554-1563, 1966. 

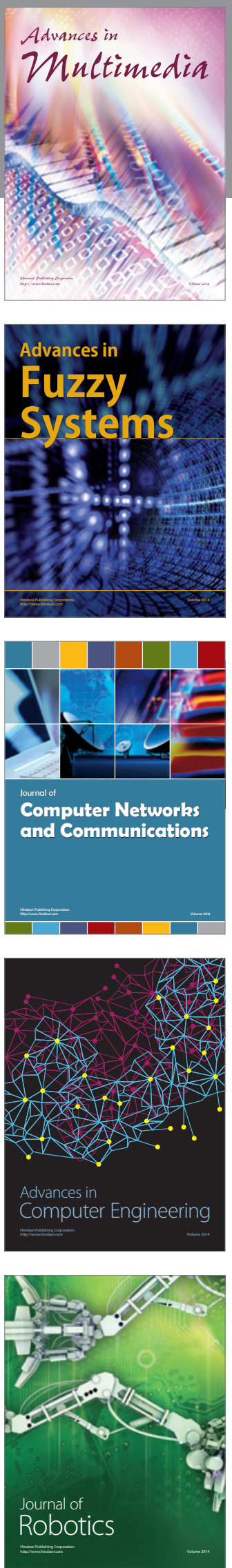

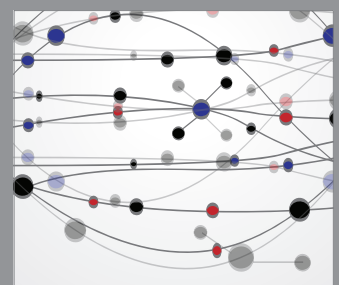

The Scientific World Journal
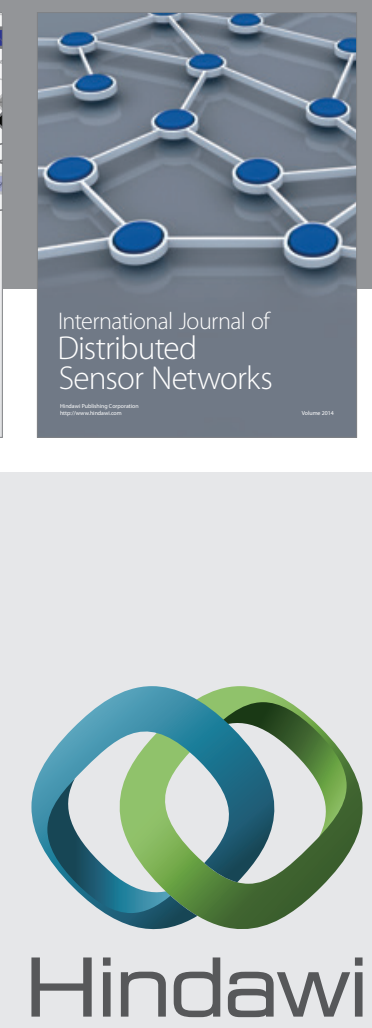

Submit your manuscripts at

http://www.hindawi.com
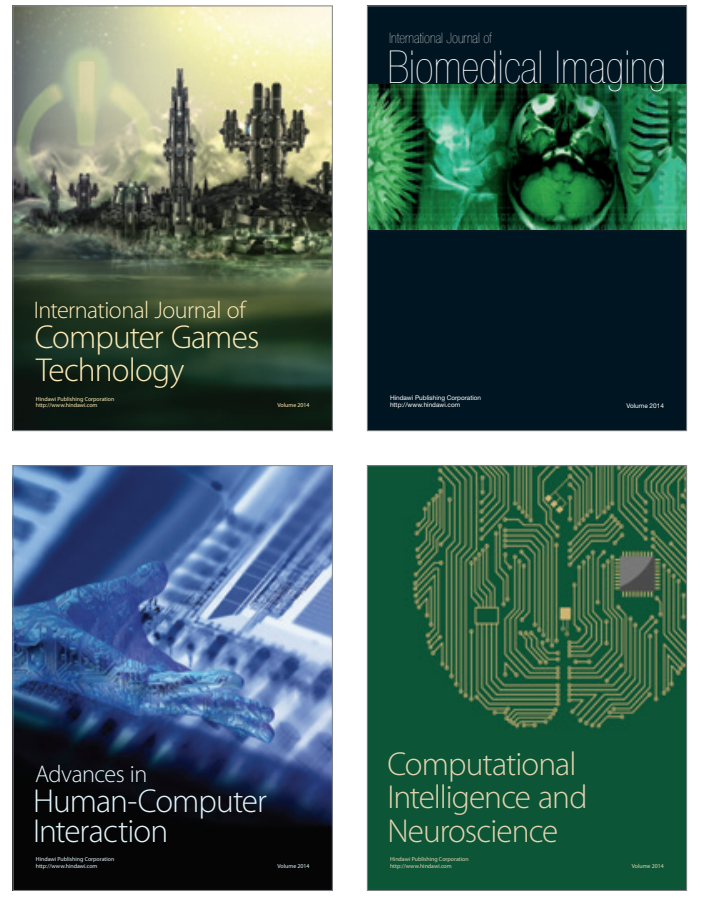
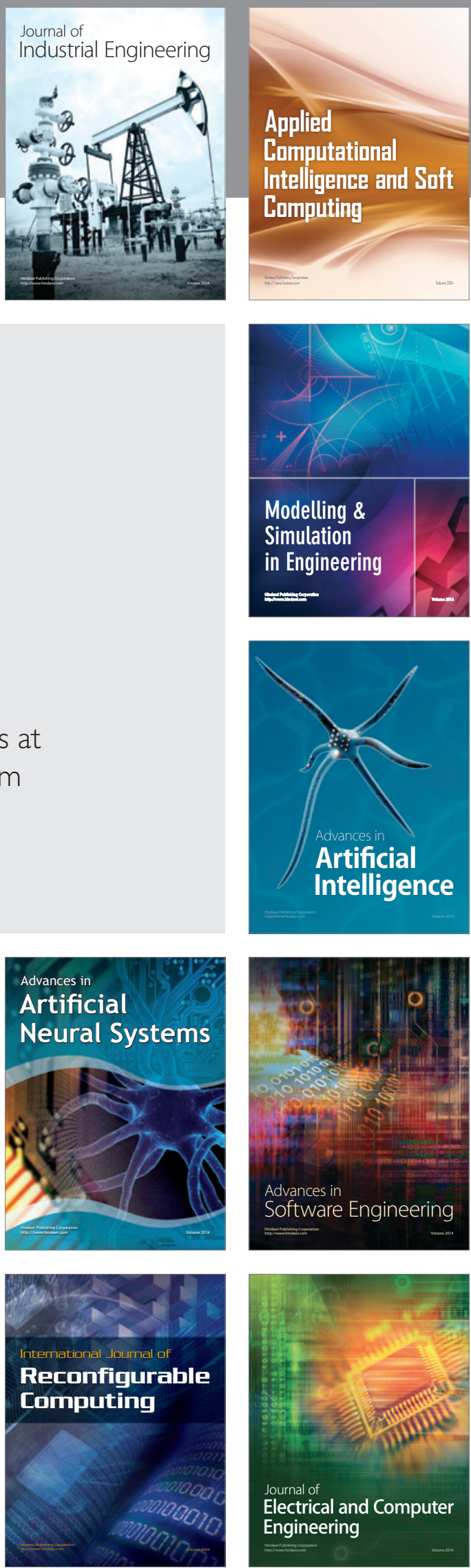